\title{
Formation of intracardiac thrombus during cardiopulmonary bypass despite full heparinization and adequate activated clotting time -A case report-
}

\author{
So Yeon Kim ${ }^{1,2}$, Jong Wook Song ${ }^{1,2}$, Yoon-Seong Jang ${ }^{1}$, and Young Lan Kwak ${ }^{1,2}$ \\ ${ }^{1}$ Department of Anesthesiology and Pain Medicine, ${ }^{2}$ Anesthesia and Pain Research Institute, Yonsei University College of Medicine, \\ Seoul, Korea
}

We reports a case of a newly formed thrombus in the left atrial appendage during cardiopulmonary bypass detected by transesophageal echocardiography in a patient with chronic atrial fibrillation and mitral stenosis. This case alerts the anesthesiologists of possible thrombus formation despite full heparinization during cardiac surgery and the importance of a comprehensive echocardiography examination. (Korean J Anesthesiol 2012; 62: 571-574)

Key Words: Atrial appendage, Atrial fibrillation, Cardiopulmonary bypass, Mitral stenosis, Thrombus, Transesophageal echocardiography.

A cardiopulmonary bypass $(\mathrm{CPB})$ is essential for valve surgery but is associated with extensive thrombin generation that cannot be prevented completely through anticoagulation with heparin $[1,2]$. We report a newly formed fresh left atrial appendage (LAA) thrombus detected during CPB under full heparinization by intraoperative transesophageal echocardiographic (TEE) monitoring in a patient with mitral stenosis (MS) undergoing a mitral valve replacement (MVR).

\section{Case Report}

A 65-year-old man, $163 \mathrm{~cm}$ in height and $60 \mathrm{~kg}$ in weight, was admitted to the emergency room with a cough, blood tinged sputum and dyspnea. He underwent percutaneous mitral balloon valvuloplasty 15 years earlier due to MS. He had a history of a cerebral infarction and hypertension. Electrocardiography revealed atrial fibrillation (A-Fib). Minimal coronary artery occlusive disease was identified during coronary angiography. Transthoracic echocardiography (TTE) revealed severe MS (mitral valve area of $0.79 \mathrm{~cm}^{2}$; mean diastolic pressure gradient of $12.5 \mathrm{mmHg}$; left atrial volume index [LAVI] of $115 \mathrm{ml} / \mathrm{m}^{2}$ ), mild tricuspid regurgitation, and a right ventricle systolic pressure of $47 \mathrm{mmHg}$ and ejection fraction (EF) of $72 \%$. Despite mild swirling in the left atrium (LA), no

Received: May 11, 2011. Revised: 1st, June 9, 2011; 2nd, June 20, 2011. Accepted: June 21, 2011.

Corresponding author: Young Lan Kwak, M.D., Ph.D., Department of Anesthesiology and Pain Medicine, Anesthesia and Pain Research Institute, Yonsei University College of Medicine, 50, Yonsei-ro, Seodaemun-gu, Seoul 120-752, Korea. Tel: 82-2-2228-8513, Fax: 82-2-364-2951, E-mail: ylkwak@yuhs.ac

(c) This is an open-access article distributed under the terms of the Creative Commons Attribution Non-Commercial License (http:// creativecommons.org/licenses/by-nc/3.0/), which permits unrestricted non-commercial use, distribution, and reproduction in any medium, provided the original work is properly cited. 
mass or thrombi were observed. The patient was medicated with digoxin, torasemide, simvastatin, and $3 \mathrm{mg}$ of warfarin. For MVR, warfarin was discontinued and heparinization was initiated four days before surgery until midnight of the day before surgery. The international normalized ratio (INR) and activated partial thromboplastin time (aPTT) one day before surgery were 1.00 and 26.3 seconds, respectively.

Before CPB, in TEE, severe spontaneous echo contrast (SEC) in the LA was noted with no evidence of thrombus at any angle (Fig. 1). CPB was instituted and after injecting 18000 units of heparin, the activated clotting time (ACT) was increased to 489 seconds. During CPB, the ACT was maintained at more than 500 seconds with an additional 3000 units of heparin. The body temperature was decreased to $33^{\circ} \mathrm{C}$. The surgeon confirmed the absence of any removable organized thrombus in the LAA and

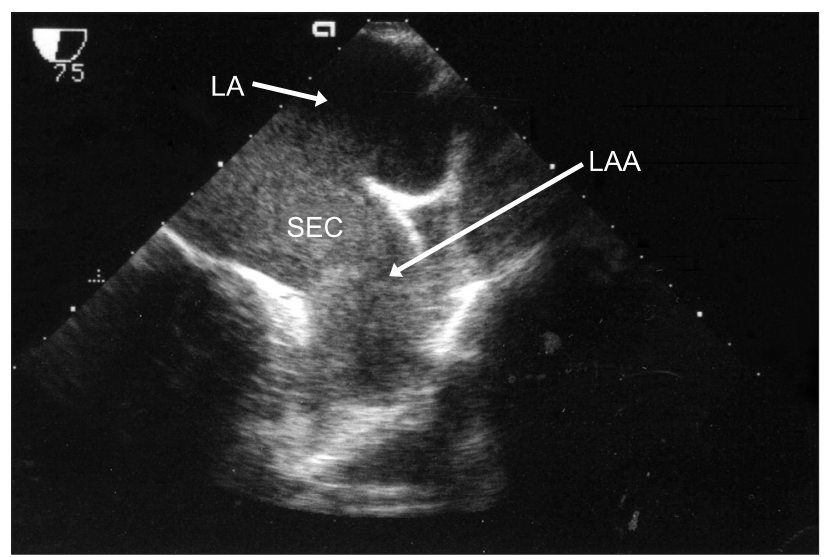

Fig. 1. Transesophageal echocardiographic, midesophageal twochamber view before the initiation of cardiopulmonary bypass. There was spontaneous echo contrast (SEC) in the left atrium (LA) without evidence of a left atrial appendage (LAA) thrombus.

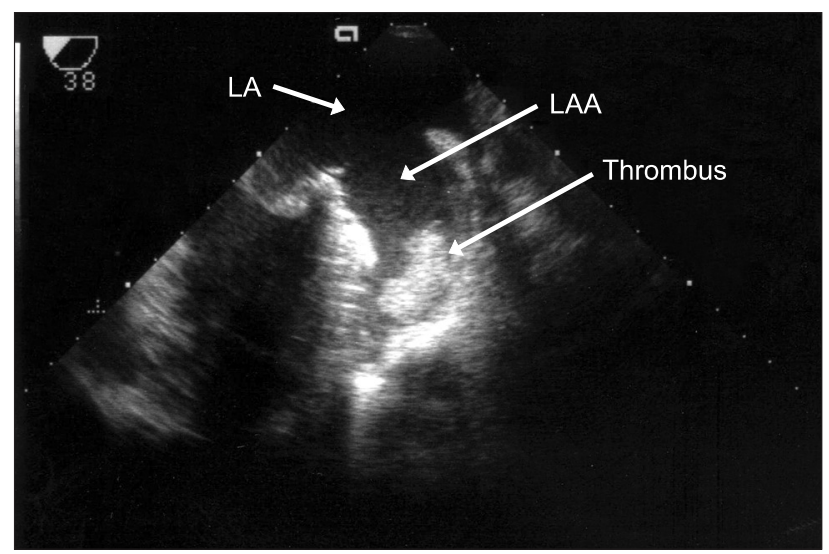

Fig. 2. Transesophageal echocardiographic, midesophageal twochamber view after mitral valve replacement during cardiopulmonary bypass. A newly formed thrombus was observed in the left atrial appendage (LAA). performed MVR with a Carpentier-Edward 27 valve.

After unclamping the aortic cross clamp (ACC), a well functioning bioprosthetic mitral valve (MV) without paravalvular leakage was confirmed through TEE. The pump flow rate was lowered to wean the patient from $\mathrm{CPB}$ and during de-airing of the heart, TEE revealed a newly formed non-mobile mass in the LAA (Fig. 2). At that time, the ACT was 569 seconds. After discussion with the surgeon, a re-bypass was planned, and the mass was removed via a left atriotomy after ACC. The clot was removed as a single piece, which measured approximately $33 \times$ $33 \mathrm{~mm}$, which had the appearance of a fresh, newly formed thrombus by a visual inspection (Fig. 3). Weaning from the CPB was performed uneventfully. The total CPB and ACC time was 81 minutes and 53 minutes, respectively.

The patient awoke six hours after arriving at the intensive care unit with no neurological deficits and was extubated at postoperative day (POD) 1. Anticoagulation was started with warfarin at POD 2. He was transferred to the general ward at POD 3 and discharged at POD 9. His postoperative TTE revealed a well functioning bioprosthetic MV, a slightly decreased LA size with a normal LV function, and no remaining MS or mitral regurgitation.

\section{Discussion}

A LA thrombus may be a common finding in patients with A-Fib, MS, an enlarged LA, advanced age, or congestive heart failure [3]. The majority of thrombi are found within the LAA, preoperatively. In patients with MS and chronic A-Fib, the severity of SEC in the LA is strongly associated with a LA thrombus and recent embolic events [4]. On the other hand, the new development of a fresh thrombus during CPB under full heparinization, as observed in this case, is extremely rare and has not been reported before. The present case had the risk factors for the development of atrial thrombus: chronic A-Fib,

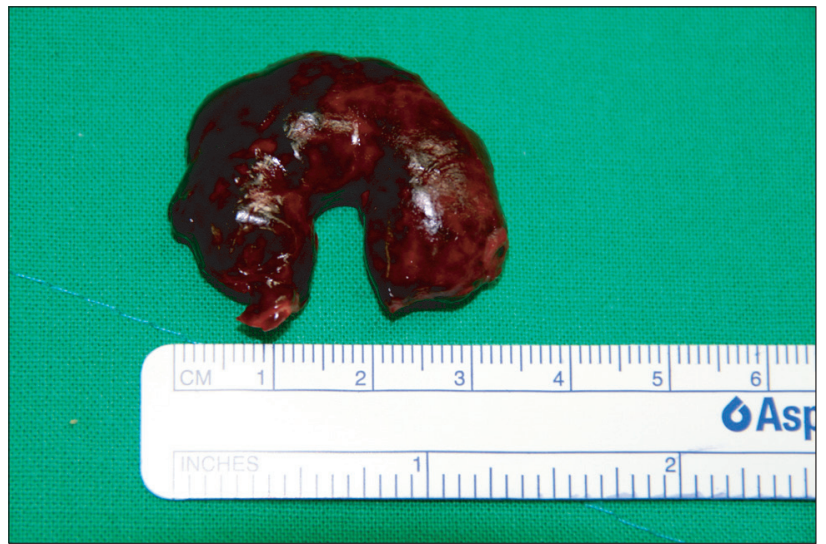

Fig. 3. Thrombus excised from the left atrial appendage. 
severe MS, enlarged LA and advanced age, but no evidence of thrombus was observed in the preoperative or intraoperative TEE before CPB. Furthermore, no organized thrombus was visible under a careful examination of the LAA by the surgeon after a left atriotomy. A visual inspection revealed the removed clot to have the appearance of a fresh, newly formed thrombus.

CPB initiates extensive thrombin generation [2], which is only suppressed partially by the use of a high dose of heparin [1]. Contact of the blood with the extracorporeal circuit via the intrinsic coagulation pathway was reported to be the main contributor to thrombin generation [2]. In addition, the retransfusion of highly thrombogenic blood aspirated from the pericardium and pleural space cavities during CPB promotes further systemic thrombin generation [5]. In the current case, it is unlikely that these etiologies contributed to thrombus formation because the pericardial blood was aspirated using a cell salvage device and was not reinfused to the patient until sternum closure.

One of the possible causes of thrombus in this case is heparin-induced thrombocytopenia (HIT), which occurs in approximately $1.9 \%$ of cardiac surgical patients [6]. The clinical features of HIT are a history of exposure to heparin, decreased platelet count of more than $50 \%$ from the baseline, and laboratory findings [7]. Although the patient in the present case used heparin during coronary angiography and after warfarin stopping before surgery, the preoperative and postoperative platelet counts were within the normal limits. Therefore, HIT may not the cause of the thrombus in the present case. Moreover, clot formation was limited to within the LAA, a feature that is different from previously reported cases of HITinduced thrombosis, which were massive and multiple in the intracavity [8] or CPB circuit [9].

After excluding HIT as the cause of the thrombus, the other possible cause is surgical trauma to the LAA. As TEE can provide false-negative results [10], it is advisable to inspect the LAA by the surgeon to confirm the absence of thrombi, particularly in patients with the above mentioned risk factors for atrial thrombi formation. This surgical trauma to the LAA could have triggered extensive thrombin generation and thrombus formation in the present case. Indeed, thrombus formation at the coronary sinus has been reported in MVR [11] and repair of the tetralogy of Fallot [12]. In these cases, surgical trauma to the coronary sinus was considered to be a possible cause of thrombosis. On the other hand, as the thrombus was detected after surgery in these cases, it is different from the present case of thrombus formed during CPB under full heparinization.

Regardless of the cause or location, a comprehensive TEE examination is still the best way of detecting intracardiac thrombi. TEE is the gold standard for the detection of LAA thrombi, with a $93-100 \%$ sensitivity and $99-100 \%$ specificity
[13]. Although TEE has high sensitivity and specificity for the detection of LAA thrombi, false-negative results can occur because the LAA is normally multi-lobed, and a thrombus may be overlooked [10].

In this case, a newly formed LAA was observed during CPB under full heparinization, maintaining an ACT $>500$ seconds by intraoperative TEE. Although the precise mechanism is unclear, this case shows that adequate ACT during CPB does not ensure the suppression of thrombus formation. This case also highlights the importance of routine serial and comprehensive intraoperative TEE during cardiac surgery because it prevented the patient from unexpected thromboembolic complications in the current case.

\section{References}

1. Brister SJ, Ofosu FA, Buchanan MR. Thrombin generation during cardiac surgery: is heparin the ideal anticoagulant? Thromb Haemost 1993; 70: 259-62.

2. Edmunds LH Jr, Colman RW. Thrombin during cardiopulmonary bypass. Ann Thorac Surg 2006; 82: 2315-22.

3. Wann LS, Curtis AB, January CT, Ellenbogen KA, Lowe JE, Estes NA 3rd, et al. 2011 ACCF/AHA/HRS focused update on the management of patients with atrial fibrillation (updating the 2006 guideline): a report of the American College of Cardiology Foundation/American Heart Association Task Force on Practice Guidelines. Circulation 2011; 123: 104-23.

4. Gonzalez-Torrecilla E, Garcia-Fernandez MA, Perez-David E, Bermejo J, Moreno M, Delcan JL. Predictors of left atrial spontaneous echo contrast and thrombi in patients with mitral stenosis and atrial fibrillation. Am J Cardiol 2000; 86: 529-34.

5. Weerwind PW, Lindhout T, Caberg NE, De Jong DS. Thrombin generation during cardiopulmonary bypass: the possible role of retransfusion of blood aspirated from the surgical field. Thromb J 2003; $1: 3$.

6. Walls JT, Curtis JJ, Silver D, Boley TM, Schmaltz RA, Nawarawong W. Heparin-induced thrombocytopenia in open heart surgical patients: sequelae of late recognition. Ann Thorac Surg 1992; 53: 787-91.

7. Follis F, Schmidt CA. Cardiopulmonary bypass in patients with heparin-induced thrombocytopenia and thrombosis. Ann Thorac Surg 2000; 70: 2173-81.

8. Legare JF, Arora R, Wood JW. Massive intracavitary clot formation during cardiopulmonary bypass. Can J Cardiol 2004; 20: 825-6.

9. Salmi L, Elalamy I, Leroy-Matheron C, Houel R, Thebert D, Duvaldestin P. Thrombosis of a cardiopulmonary bypass circuit despite recommended hypocoagulation with danaparoid during the acute phase of type II heparin-induced thrombocytopenia. Ann Fr Anesth Reanim 2006; 25: 1144-8.

10. Tanzola RC, Milne B, Hamilton A. Transesophageal echocardiography of a dislodged left atrial appendage thrombus and its subsequent surgical removal during coronary artery bypass graft surgery. J Am Soc Echocardiogr 2010; 23: 1008 e1-3.

11. Hazan MB, Byrnes DA, Elmquist TH, Mazzara JT. Angiographic 
demonstration of coronary sinus thrombosis: a potential consequence of trauma to the coronary sinus. Cathet Cardiovasc Diagn 1982; 8: 405-8.

12. Bapat VN, Hardikar AA, Porwal MM, Agrawal NB, Tendolkar AG. Coronary sinus thrombosis after cannulation during cardio- pulmonary bypass. Ann Thorac Surg 1996; 62: 1506-7.

13. Manning WJ, Weintraub RM, Waksmonski CA, Haering JM, Rooney PS, Maslow AD, et al. Accuracy of transesophageal echocardiography for identifying left atrial thrombi. A prospective, intraoperative study. Ann Intern Med 1995; 123: 817-22. 